Ophthalmologe 2022 $\cdot 119: 801-806$ https://doi.org/10.1007/s00347-022-01577-8 Eingegangen: 17. Dezember 2021 Überarbeitet: 3. Januar 2022 Angenommen: 7. Januar 2022 Online publiziert: 25. Januar 2022 (๑) Der/die Autor(en) 2022

\section{Regression einer idiopathischen epiretinalen Gliose - Kasuistik und Überlegungen zum Mechanismus}

\author{
C. Framme 1 (D) N. Feltgen ${ }^{2} \cdot$ R. Schumann 3 - D. O. Stichtenoth ${ }^{4} \cdot$ L. O. Hattenbach ${ }^{5}$ \\ ${ }^{1}$ Universitäts-Augenklinik, Medizinische Hochschule Hannover, Hannover, Deutschland \\ ${ }^{2}$ Universitäts-Augenklinik, Universitätsmedizin Göttingen, Göttingen, Deutschland \\ ${ }^{3}$ Universitäts-Augenklinik, Ludwig-Maximilians-Universität München, München, Deutschland \\ ${ }^{4}$ Institut für Klinische Pharmakologie, Medizinische Hochschule Hannover, Hannover, Deutschland \\ ${ }^{5}$ Augenklinik, Klinikum der Stadt Ludwigshafen gGmbH, Ludwigshafen am Rhein, Deutschland
}

\title{
Zusammenfassung
}

Bei Vorliegen einer symptomatischen epiretinalen Gliose wird im klinischen Alltag in der Regel eine Pars-plana-Vitrektomie mit Membranpeeling zur Entfernung der Membran indiziert. Nach gängiger klinischer Erfahrung existiert nahezu keine selbstständige Regression einer solchen epiretinalen Membran und somit einer alleinigen Abheilung der Pathologie. Beschrieben wird daher der ungewöhnliche Fall einer beidseitigen selbstständigen Regression einer idiopathischen epiretinalen Gliose mit Makulaschichtforamen bei einem 73-jährigen Patienten. Überlegungen zum möglichen Mechanismus werden auf Basis der vorhandenen Literatur vorgestellt. Diese beinhalten die Trennung entzündlicher vs. nichtentzündlicher Membranen, mögliche Separation einzelner Schichten in Abhängigkeit vom Status der hinteren Glaskörpergrenzmembran, aber auch das mögliche Wirken proteolytischer Systeme im Bereich des hinteren Glaskörpers. Es stellt sich schließlich die Frage, ob Patienten vor möglicher Operation über diesen Umstand mit aufgeklärt werden müssen.

\section{Schlüsselwörter}

Epiretinale Gliose · Makulaschichtforamen · Glaskörperabhebung · Vitrektomie · SD-OCT

Eine epiretinale Gliose ist eine Diagnose, die bei entsprechender Symptomatik wie Visusverlust und/oder Metamorphopsie eine typische chirurgische Indikation zur Entfernung darstellt [1]. Über die moderne Schnittbilddiagnostik mittels SpectralDomain-optische Kohärenztomographie (SD-OCT) sind die Membranen heutzutage detailliert darstellbar. Die alleinige Präsenz einer solchen Membran ist allerdings kein Grund, eine sofortige chirurgische Intervention zu indizieren. Häufig ergeben sich für die Patienten mit dieser Diagnose (noch) keine Sehstörungen. Darüber hinaus kann eine epiretinale Membran - beispielsweise ohne signifikante vitreomakuläre Traktion - über sehr lange Zeiten nahezu unverändert und stabil existieren. In solchen Fällen einer „stummen“ epiretinalen Gliose sollte nicht operiert und der Patient über die "Gutartigkeit" seiner Diagnose aufgeklärt werden. Häufig können Gliosen auch mit einem makulären Schichtforamen assoziiert sein, das - insbesondere bei fehlender Metamorphopsie - eher keine Indikation zur Operation darstellt, da der Visus in der Regel recht gut ist und bleibt und eine entsprechende Vitrektomie mit Peeling hier häufig keinen Benefit erbringt [2].

Im Falle einer klinisch operationswürdigen Gliose kann der "richtige" Zeitpunkt der Intervention diskutiert werden, in der Regel gilt aber, dass eine Operation dann angezeigt ist, wenn der Patient sich gestört fühlt und einen entsprechenden Leidens- 

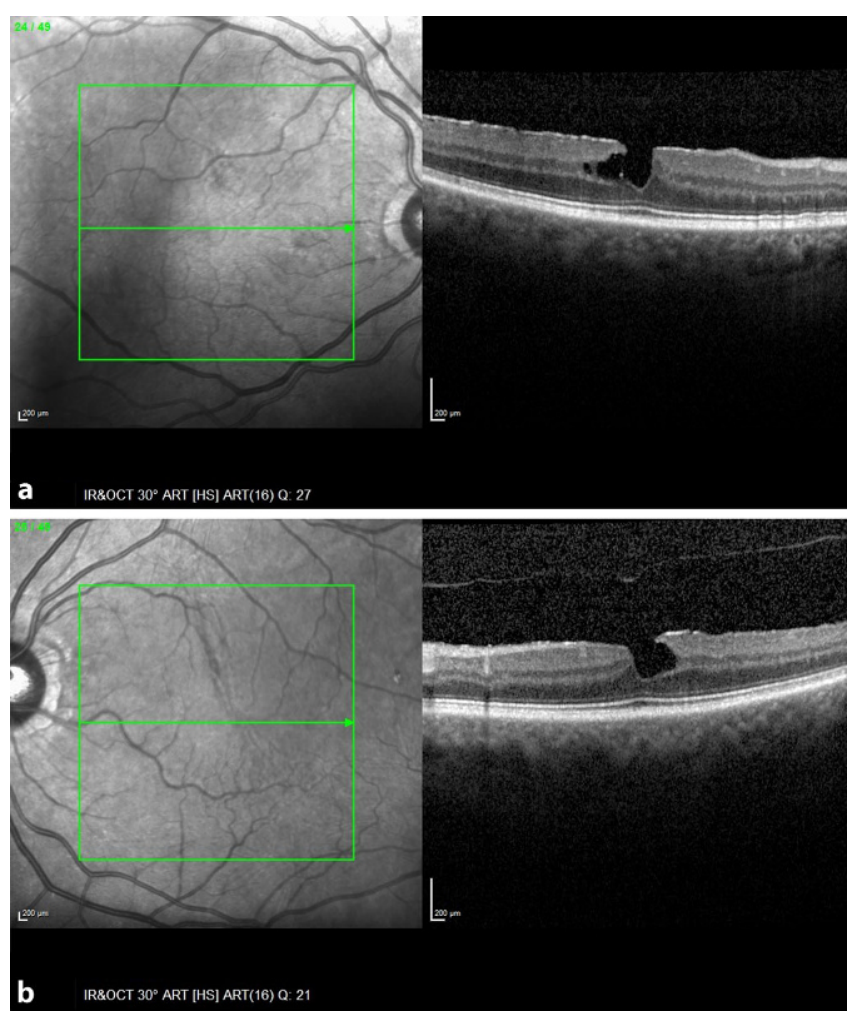

Abb. $1 \triangleleft$ a, bSD-

OCT des rechten (a) und linken Auges (b). Beidseits imponieren eine epiretinale Gliose rechts stärker als links sowie ein assoziiertes Makulaschichtforamen. Zumindest für das linke Auge kann anhand der Bilder die Aussage getroffen werden, dass die hintere Glaskörpergrenzmembran dem hinteren Pol noch anliegt, ohne allerdings mit der Makula mechanisch verbunden zu sein

druck entwickelt. Häufig wird man seitens des Patienten gefragt, ob es alternative Behandlungsmöglichkeiten zur Operation gibt oder ob sich die Membran ggf. sogar von selbst zurückbilden kann. Ehrlicherweise haben die Autoren bis zum Auftreten des dargestellten Falles auf diese Frage geantwortet, dass sich eine epiretinale Gliose nicht von alleine zurückbildet und bei Auftreten von Sehbeschwerden in der Regel eine chirurgische Intervention erfolgen sollte. Man darf davon ausgehen, dass das auch die Regel bleibt, dennoch kann sich eine idiopathische epiretinale Gliose erstaunlicherweise selbstständig zurückbilden, wie dieser Fall nahelegt.

\section{Fallbeschreibung}

Ein 73-jähriger männlicher Patient stellte sich im Jahr 2016 erstmals mit der Frage einer möglichen Kataraktoperation vor. Sehprobleme wie beispielsweise Metamorphopsie wurden verneint, vorherige Augenoperationen bestanden nicht, es wurden allerdings Latanoprost-Augentropfen beidseits appliziert, wobei die Familienanamnese für Glaukom negativ war und der anamnestisch jemals maximal gemessene Augeninnendruck $25 \mathrm{~mm} \mathrm{Hg}$ betrug.

Die umfassende Untersuchung ergab einen Visus rechts von 1,2 (+2,25sph; $-0,25 \mathrm{cyl}, 125^{\circ}$ ) und links von 0,8 ( $+3,25 \mathrm{sph}$; $-0,5 \mathrm{cyl} 87^{\circ}$ ). Die applanatorisch gemessene Tensio betrug $20 \mathrm{~mm} \mathrm{Hg}$ beidseits. In der Spaltlampenuntersuchung zeigte sich eine milde und reizfreie Cataracta corticalis et nuclearis beidseits. Zeichen eines Pigmentdispersionsglaukoms oder einer Pseudoexfoliation ergaben sich nicht. Fundoskopisch imponierte beidseits eine vitale und randscharfe Papille ohne signifikante Exkavation, die periphere Netzhaut lag an, der Gefäßstatus war regelrecht, und im Makulabereich konnte minimal das typische Glitzern einer epiretinalen Gliose wahrgenommen werden. Das Gesichtsfeld war beidseits frei und die SD-OCT der Papille ergab beidseits regelrecht innerhalb der Norm liegende Nervenfaserschichtdicken. Die SD-OCT der Makula zeigte beidseits die bereits fundoskopisch vermutete Gliose und zusätzlich ein beidseitiges Makulaschichtforamen (- Abb. 1).

Wir stellten somit oben genannte Diagnose der entsprechenden Makulaveränderung und empfahlen bei fehlenden Seh- beschwerden und nicht vorhandenem Leidensdruck zunächst abzuwarten, klärten über die Gutartigkeit des Befundes und die vorhandene Chance einer Langzeitstabilität auf. Ebenfalls wurde auf die nicht vorhandenen Befunde einer möglichen Glaukomerkrankung hingewiesen und ggf. sogar ein Absetzen der Augentropfen diskutiert. Eine Wiedervorstellung nach 1 Jahr wurde vorgeschlagen.

Bei weiterhin fehlendem Leidensdruck stellte sich der Patient erst nach 3,5 Jahren wieder bei uns vor. Die drucksenkende Augentropfentherapie wurde über die Zeit weiterhin eingenommen, Operationen oder anderweitige Augenbehandlungen wurden nicht durchgeführt. Bei der klinischen Untersuchung betrug der Visus rechts 1,0 und links 1,2 mit Korrektur. Augeninnendruck und vordere Augenabschnitte waren weiterhin unauffällig, die SD-OCT der Nervenfaserschichtdicken regulär. Fundoskopisch zeigten sich die Papillen vital und randscharf, die Netzhaut lag zirkulär an. Bei fehlender Fundusfotografie kann nicht objektiv beurteilt werden, ob sich das gliotische Glitzern reduziert hat, zumindest zeigte aber die durchgeführte makuläre SD-OCT beidseits, dass sowohl die Gliose als auch die Makulaschichtforamina nicht mehr vorhanden waren (-Abb. 2). Beidseits imponierte nun eine nahezu reguläre foveale Senke ohne weitere Pathologien.

Der Patient wurde nochmals 1 Jahr später untersucht, wobei sich prinzipiell exakt gleiche Befunde ergaben. Die epiretinale Gliose ist an beiden Augen ohne irgendeine Intervention von selbst in Regression gegangen. Dem Patienten wurden weitere Kontrollen bei Bedarf anheimgestellt.

\section{Diskussion}

In der Literatur sind zur Thematik der Regression von epiretinalen Gliosen relativ wenige und durchaus auch wechselhafte Information zu finden, allerdings erscheint eine solche Membranregression offensichtlich häufiger vorzukommen als vermutet. Das mag erstaunen, da sich ein solcher Fall im klinischen Alltag nach bisheriger Einschätzung des Erstautors eigentlich nicht wirklich darstellt. Was aber genau zeigen diese Publikationen? Eine aktuelle Arbeit beschreibt eine entsprechen- 

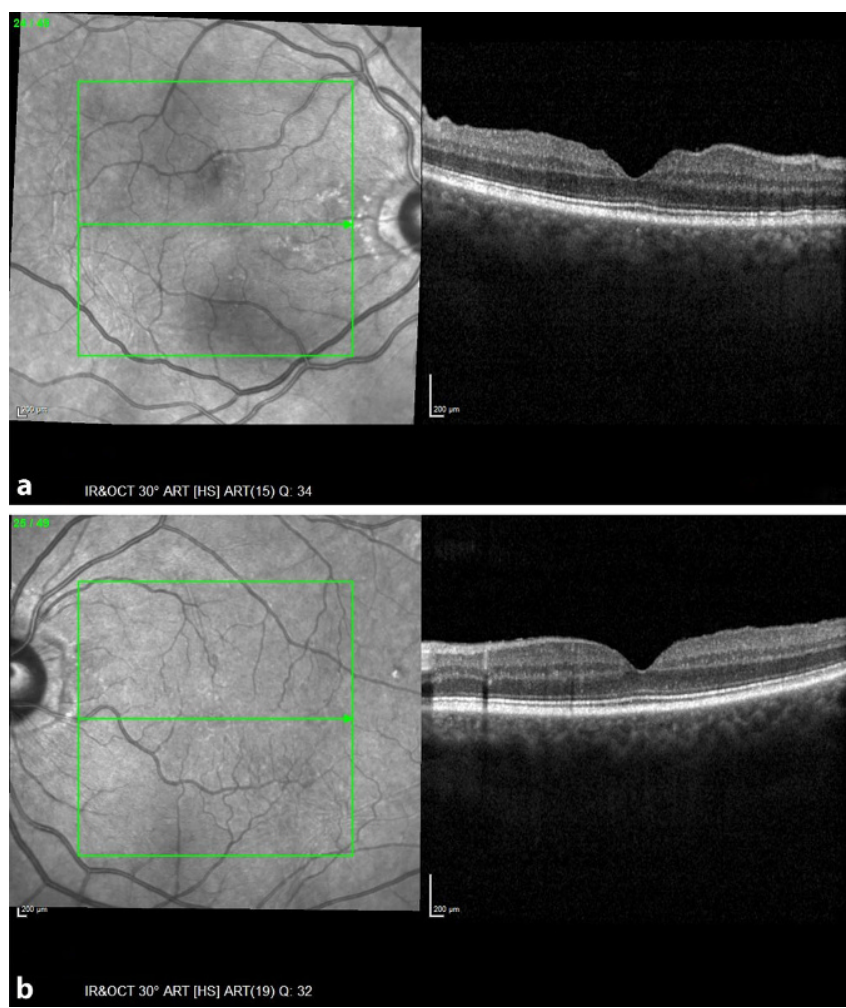

Abb. $2 \triangleleft$ a, b SD-

OCT des rechten (a) und linken Auges (b) ca. 3,5 Jahre nach der ersten Untersuchung und ohne jegliche Intervention in der Zwischenzeit. Gliose und Makulaschichtforamen sind nicht mehr nachweisbar, und es zeigt sich beidseits eine nahezu reguläre foveale Konfiguration. Der linke Glaskörper hat sich offensichtlich vom hinteren Pol abgelöst, die Grenzmembran ist im OCT nicht mehr sichtbar

de Regression einer entzündlichen Membran in 3 uveitischen Augen [3], wobei es sich im ersten der dargestellten Fälle eher um eine posteriore Glaskörperabhebung mit verdickter Grenzmembran handelt, im zweiten Fall eine vitreomakuläre Traktion vorhanden zu sein scheint und im dritten Fall eine lediglich partielle Regression der Membran beschrieben wird [3]. Andere Autoren widmen sich ebenfalls diesem Phänomen und sehen spontane Auflösungen oder Separationen epiretinaler Membranen bei Atrophie des Sehnerven [4] oder gerade auch bei sehr jungen Patienten bis ca. 30 Jahre [5]. In letztgenannter Publikation wird von idiopathischen Membranen berichtet, die sich separieren (offensichtlich nicht auflösen) und die von den Autoren als "immatur" klassifiziert werden, wobei - so wird es diskutiert die eigene Kontraktionskraft innerhalb dieser Membranen stärker zu sein scheint als die retinale Anheftungskraft, ein Umstand der dadurch zur Abhebung der Membran führt [5]. Histologien von geneigten Fällen sollen zeigen, dass gerade die idiopathischen epiretinalen Membranen RPE-Zellen enthalten, was Membranen inflammatorischer Genese nicht tun [6], sodass es sich bei den Membranen um unterschiedliche Entitäten handelt.

Zu vermuten wäre nun, dass sich eine Regression vielleicht eher bei inflammatorischen Membranen zeigt, da der klinische Alltag - wie oben beschrieben - in der Regel eher eine Progression oder eine Stabilität von idiopathischen Membranen aufzeigt. Nun verweisen Guemes-Villahoz et al. [3] allerdings darauf, dass eine spontane Auflösung der epiretinalen Membran eher bei idiopathischen als bei inflammatorischen Fällen auftritt, und zitieren dabei insbesondere die Arbeit von Kida et al. [7], Nomoto et al. [8] und Yang et al. [9]. Kida et al. fanden von $n=604$ Patienten mit epiretinaler Membran bei $n=14$ eine spontane Separation („release ${ }^{\prime \prime}$ [7]. Ein schöner Fall dieser Publikation zeigt eine partielle Abschilferung der Membran im SD-OCT (, auto-peeling ${ }^{\prime \prime}$ ), wobei diese recht prominente Membran aber nicht verschwindet [7]. In der Arbeit von Nomoto et al. aus dem Jahr 2013 wird die Separation maßgeblich über die schriftliche Dokumentation von Metamorphopsien anstatt über Fotodokumentation nachgewiesen [8], was eher auf eine unzureichende Nachweistechnik hindeuten mag. Auch Yang et al. beschreiben im Wesentlichen Separatio- nen der Membranen und legen hier sinnvollerweise den Fokus auf den Status der posterioren Glaskörpergrenzmembran [9]. Bei über 1200 Fällen von idiopathischen epiretinalen Membranen fanden die Autoren hier in einem durchschnittlichen Zeitraum von etwa 33 Monaten eine spontane Separation der Membran in 1,5\% der Fälle, wenn der Glaskörper abgehoben war, und in 13,4\% der Fälle, wenn der Glaskörper noch anliegend war [9]. Der Status der Glaskörperanlage am hinteren Pol scheint damit in der Tat nachvollziehbar ein wichtiger Faktor für eine mögliche Separation zu sein.

Für uns stellt sich damit allerdings dann die Frage, ob man bei anliegendem Glaskörper überhaupt eine eigenständige epiretinale Membran von einer etwaigen hinteren Glaskörperverdichtung (ohne Traktion wie etwa beim vitreomakulären Traktionssyndrom) unterscheiden kann. Schaut man sich die SD-OCTs unseres Patienten an, so ist zumindest am linken Auge klar zu erkennen, dass der hintere Glaskörper im zentralen Bereich der Makula abgehoben ist und keine Adhäsion zur Netzhautoberfläche erkennen lässt. Eine mechanische Beeinflussung der Gliose durch den Glaskörper erscheint somit nicht möglich. Dennoch ist - davon ist stets auszugehen, wenn die Glaskörpergrenzmembran im SD-OCT zu sehen ist - der hintere Glaskörper in der Regel noch am hinteren Pol anliegend. Dieses konnte auch durch die SD-OCT des Papillenrandes bestätigt werden. In der Verlaufskontrolle hat sich der Glaskörper dann offensichtlich deutlich - wahrscheinlich komplett vom hinteren Pol abgelöst, und die epiretinale Gliose ist ebenfalls verschwunden. Die vorher gut sichtbare Glaskörpergrenzmembran war im SD-OCT nicht mehr vorhanden. Da es aber auch im Vorfeld keine Verbindung zwischen beiden Grenzfächen gab, ist unklar, ob hier überhaupt ein mechanischer Trigger vorlag oder ob die Membran beispielsweise andersartig abgebaut wurde (dann eher keine Separation?) und in Folge auch die Schichtforamina abheilen konnten.

In der Tat wäre die einfache Auflösung der Membran auch durch beispielsweise enzymatischen Abbau denkbar. Der hintere Augenabschnitt verfügt nämlich, wie bereits sehr lange bekannt ist, über ein 
recht gut funktionierendes, über Plasminogenaktivatoren und -inhibitoren gesteuertes Fibrinolysesystem, das nicht nur für die Auflösung von Blutungen, sondern auch für die Matrixdegradation eine wichtige Rolle spielt [10-12]. Eine mögliche Hypothese für das „Verschwinden" der Membran wäre somit also auch der Gewebeplasminogenaktivator-vermittelte Abbau von Gewebematrix durch das intraokuläre körpereigene Fibrinolysesystem mit oder ohne vorheriges Lösen der Membran durch Separation.

Im Gegensatz zu den vorgestellten Literaturbeispielen scheint in unserem Fall somit eben keine einfache "Separation" der Membran über die Glaskörpergrenzmembran vorzuliegen, sondern eher eine wie auch immer geartete „Auflösung“ zumindest in den relevanten zentralen Makulabereichen. Die Übersichtsaufnahmen der SD-OCT zeigen nämlich darüber hinaus, dass in der peripheren Makula ja durchaus noch Membranreste existieren, die Membran sich somit also nicht in Gänze separiert haben kann.

Auf Basis unserer hier durchgeführten, eher eingeschränkten Bildgebung und des relativ langen Zeitraums zwischen Diagnose und Regression der Membran kann leider in der Tat keine eindeutige Aussage zum definitiven Mechanismus dieses positiven Verlaufes für den Patienten getätigt werden. Die Überlegungen dazu sind somit spekulativer Natur. Denkbar sind diesbezüglich neben dem bereits oben diskutierten enzymatischen Abbau auch unter dem Aspekt der Vitreoschisis zeitlich unterschiedliche Separationen einzelner Glaskörperschichten im Rahmen der spontanen Lösung der vitreopapillären Adhäsion. Multicoloraufnahmen wären evtl. in der Lage gewesen, eine genauere Beurteilung der Zugkräfte der epiretinalen Membran zu ermöglichen. Eine Änderung des Zugvektors und der Traktionskraft könnten bereits für die Befundbesserung entscheidend sein, dieses müsste in diesem Fall dann allerdings eher unabhängig von einer mechanischen Beeinflussung des Glaskörpers passiert sein. Auch wäre bei beginnenden Makulaschichtforamina ein zusätzlicher Stern-Scan in der OCT zielführend, um die zentrale Pathologie besser darstellen zu können und ggf. dadurch weitere Rückschlüsse ziehen zu können.
Nichtsdestotrotz zeigt aber auch die vorhandene OCT-Bildgebung in unserem Fall die eindeutige und beidseitige Regression der Membran mit schöner Abheilung der Makulaschichtforamina zum Vorteil des Patienten.

Ein weiterer interessanter Aspekt in dieser Diskussion ergab sich dankenswerter Weise über den Reviewer dieses Artikels im Einreichprozess, der darauf verwies, dass auch er einen ähnlichen Fall von Membranregression unter Einnahme von Latanoprost - wie das auch hier als Dauermedikation vorlag - beobachten konnte. Die Literaturrecherche nach einem möglichen pharmakologischen Zusammenhang ergab dabei aber eher ein uneinheitliches Ergebnis. Offensichtlich existieren keine zell- oder tierexperimentellen Daten oder gar klinische Nachweise zu einem möglichen Zusammenhang zwischen Latanoprost (Prostaglandinanalogon PGF2alpha) und einer möglichen Membranregression. Indirekte Hinweise in einem aktuellen Review aus dem Jahr 2021 beschreiben für das PGF2alpha eine eher proaktive Kraft zur Fibrosebildung [13], während hingegen ältere Arbeiten aus den späten 90erJahren für PGF2alpha als auch seinem Analogon Latanoprost in Zellkulturen eine Induktion von Metalloproteinasen, eine Verminderung von Kollagen und anderer Bestandteile extrazellulärer Matrix finden, allerdings im Bereich der Muskelzellen des Ziliarkörpers [14-16]. Da in idiopathischen epiretinalen Membranen aber neben Hyalozyten und Müller-Zellen auch Myofibroblasten mit kontraktilen Eigenschaften gefunden werden [17], könnten zukünftige Versuche der medikamentösen Degradation gliotischen Gewebes mittels Latanoprost vielleicht interessant sein.

Insgesamt halten wir den hier dargestellten Fall für besonders, da eine in der SD-OCT doch deutlich darzustellende makuläre Pathologie mit eher milder Gliose (ohne Traktion!), aber deutlicher Schichtforamenbildung einfach ohne chirurgische Intervention in eine wie auch immer geartete Regression übergegangen ist. Dieses erscheint klinisch eher ungewöhnlich und offensichtlich nur sehr selten der Fall zu sein, wird in der Literatur aber durchaus in verschiedenen Formen beschrieben, wobei uns der genaue Mechanismus, wie oben diskutiert, fraglich erscheint und un- terschiedlich sein kann. Insbesondere ist nicht klar, ob die in der Literatur gebräuchlichen Termini „Regression“ und "Separation" verschiedene Mechanismen - ggf. in Abhängigkeit des Glaskörperstatus - andeuten sollen.

Das abwartende Verhalten (keine Indikation zur Operation!) bei unserem Patienten war aufgrund der subjektiven Symptomlosigkeit der Pathologie sinnvoll, gerechnet wurde allerdings von ärztlicher Seite nicht mit einer quasi kompletten Auflösung der zentralen Pathologie, sodass dem Patienten in erster Linie mögliche Stabilität prognostiziert wurde. Gleichermaßen wurde erst kürzlich in der internationalen Literatur ein ähnlicher Fall einer einseitigen "Separation“ und das Verschwinden des Makulaschichtforamens beschrieben, in dem die Operation bereits geplant war [18]. Im OCT-Bild der Publikation ist allerdings nicht der Status des Glaskörpers mit und ohne Gliose erkennbar. Dieses ist in unserem Fall hingegen deutlich nachvollziehbar, wo der Glaskörper zwar beidseits noch dem hinteren Pol anlag, dieser allerdings - sehr gut am linken Auge zu erkennen - keinen offensichtlichen Kontakt mehr zur Netzhautoberfläche im Makulabereich und der Membran hatte, sodass man annehmen könnte, dass eben auch keine mechanische Separation zumindest glaskörperbedingt mehr möglich war.

Geht man neben den oben vorgestellten hypothetischen Überlegungen zum möglichen Mechanismus der Membranregression davon aus, dass der wichtigste Trigger am ehesten doch die Glaskörperabhebung sein mag (s. auch [9]), so stellt sich die Frage, ob man Patienten im klinischen Alltag auch darüber aufklären sollte, dass die Pathologie der epiretinalen Gliose durchaus auch von selbst verschwinden kann (immerhin geht es um die Frage chirurgischer Intervention!). Da dieses nach Ansicht der Autoren aber offensichtlich doch so extrem selten ist, erscheint das wahrscheinlich nicht zielführend. Letztlich könnte aber eine solche Frage theoretisch von dem Befund in der SD-OCT und der vorhandenen oder auch nicht vorhandenen Glaskörperabhebung abhängig gemacht werden, was eben auch eine vielleicht noch genauere Diagnostik bedingen würde, insbesondere wenn der Glaskörperstatus in der OCT 
nicht zu erheben wäre. Das hieße dann, dass bei nachweisbar noch anliegendem Glaskörper trotz Symptomen eher noch ein abwartendes Verhalten gerechtfertigt wäre (insbesondere, wenn zusätzlich ein Makulaschichtforamen vorliegt, wie dieses auch in [18] empfohlen wird), dass aber ein definitiv abgehobener Glaskörper kein weiteres Abwarten auf mögliche Regression rechtfertigen würde. Sicherlich müsste das allerdings, wie bereits gehandhabt, immer vom Einzelfall abhängig entschieden werden. Eine sehr ausführliche und lesenswerte Stellungnahme zur Diagnostik und Therapie der epiretinalen Gliose liegt seitens der DOG, der RG und des BVA von 2021 [19] vor, nach der man sich bezüglich dieses Krankheitsbildes gut richten kann. Interessanterweise findet sich in dieser Publikation allerdings keine Erläuterung zur möglichen selbstständigen Auflösung und somit zum spontanen Verschwinden der Membran, was nochmals den Seltenheitswert dieses Kasus unterstreichen mag.

\section{Korrespondenzadresse}

\section{Prof. C. Framme, MD, FEBO, MBA}

Universitäts-Augenklinik, Medizinische

Hochschule Hannover

Hannover, Deutschland

framme.carsten@mh-hannover.de

Funding. Open Access funding enabled and organized by Projekt DEAL.

\section{Einhaltung ethischer Richtlinien}

Interessenkonflikt. C. Framme, N. Feltgen, R. Schumann, D.O. Stichtenoth und L.O. Hattenbach geben an dass kein Interessenkonflikt besteht.

Für diesen Beitrag wurden von den Autoren keine Studien an Menschen oder Tieren durchgeführt. Für die aufgeführten Studien gelten die jeweils dort angegebenen ethischen Richtlinien.

Open Access. Dieser Artikel wird unter der Creative Commons Namensnennung 4.0 International Lizenz veröffentlicht, welche die Nutzung, Vervielfältigung, Bearbeitung, Verbreitung und Wiedergabe in jeglichem Medium und Format erlaubt, sofern Sie den/die ursprünglichen Autor(en) und die Quelle ordnungsgemäß nennen, einen Link zur Creative Commons Lizenz beifügen und angeben, ob Änderungen vorgenommen wurden.

Die in diesem Artikel enthaltenen Bilder und sonstiges Drittmaterial unterliegen ebenfalls der genannten Creative Commons Lizenz, sofern sich aus der Abbil-

\section{Regression of idiopathic epiretinal membrane-Case report and consideration of the possible mechanism}

In the presence of a symptomatic epiretinal gliosis, pars plana vitrectomy with membrane peeling to remove the membrane is usually indicated in clinical practice. According to common clinical experience, almost no independent regression of such an epiretinal membrane and thus healing of the pathology alone exists. Therefore, the unusual case of bilateral independent regression of idiopathic epiretinal gliosis and formation of a lamellar macular hole in a 73-year-old male patient is described. Considerations of the possible mechanism are presented based on the existing literature. These include separation of inflammatory versus noninflammatory membranes, possible separation of individual layers depending on the status of the posterior vitreous limiting membrane and also the possible action of proteolytic systems in the posterior vitreous region. Finally, the question arises, whether patients have to be informed about this fact before possible surgery.

\section{Keywords}

Epiretinal membrane $\cdot$ Lamellar macular hole $\cdot$ Vitreous detachment · Vitrectomy · SD-OCT

dungslegende nichts anderes ergibt. Sofern das betreffende Material nicht unter der genannten Creative Commons Lizenz steht und die betreffende Handlung nicht nach gesetzlichen Vorschriften erlaubt ist, ist für die oben aufgeführten Weiterverwendungen des Materials die Einwilligung des jeweiligen Rechteinhabers einzuholen.

Weitere Details zur Lizenz entnehmen Sie bitte der Lizenzinformation auf http://creativecommons.org/ licenses/by/4.0/deed.de.

\section{Literatur}

1. Chua PY, Sandinha MT, Steel DH (2021) Idiopathic epiretinal membrane: progression and timing of surgery. Eye (Lond). https://doi.org/10.1038/ s41433-021-01681-0

2. Purtskhvanidze K, Balken L, Hamann T, Wöster L, von der Burchard C, Roider J, Treumer F (2018) Long-term follow-up oflamellarmacular holes and pseudoholes over at least 5 years. Graefes Arch Clin Exp Ophthalmol 256(6):1067-1078. https://doi. org/10.1007/s00417-018-3972-2

3. Guemes-Villahoz N et al (2021) Spontaneous resolution of inflammatory epiretinal membrane: case series and review of the literature. Eur J Ophthalmol. https://doi.org/10.1177/ 11206721211036291

4. Wetzel B et al (2016) Regression of epiretinale membrane after optic nerve atrophy. Eur J Ophthalmol 26:e165-e167

5. Meyer $\mathrm{CH}$, Rodrigues EB, Mennel S, Schmidt JC, KrollP (2004) Spontaneous separation of epiretinal membrane in young subjects: personal observations and review of the literature. Graefes Arch Clin Exp Ophthalmol 242(12):977-985

6. Sheybani A, Harocopos GJ, Rao PK (2012) Immunohistochemical study of epiretinal membranes in patients with uveitis. J Ophthalmic Inflamm Infect 2:243-248

7. Kida T, Morishita S, Fukumoto M et al (2017) Longterm evaluation of spontaneous release of epiretinal membrane and its possible pathogenesis. Clin Ophthalmol 11:1607-1610
8. Nomoto H, Matsumoto C, Arimura E et al (2013) Quantification of changes in metamorphopsia and retinal contraction in eyes with spontaneous separation of idiopathic epiretinale membrane. Eye (Lond) 27:924-930

9. Yang HS, Hong JW, Kim YJ et al (2014) Characteristics of spontaneous idiopathic epiretinal membrane separation in spectral domain optical coherence tomography. Retina 34:2079-2087

10. Immonen I, Vaheri A, Tommila P, Sirén V (1996) Plasminogen activation in epiretinal membranes. Graefes Arch Clin Exp Ophthalmol 234(11):664-669

11. Hattenbach LO, Allers A, Gümbel HO, Scharrer I, Koch FH (1999) Vitreous concentrations of TPA and plasminogen activator inhibitor are associated with VEGF in proliferative diabetic vitreoretinopathy. Retina 19(5):383-389

12. Schacke W, Beck KF, Pfeilschifter J, Koch F, Hattenbach LO (2002) Modulation o of tissue plasminogen activator and plasminogen activator inhibitor- 1 by transforming growth factor-beta in human retinal glial cells. Invest Ophthalmol Vis Sci 43(8):2799-2805

13. Li K, Zhao J, Wang M, Niu L, Wang Y, Li Y, Zheng Y (2021) The roles of various prostaglandins in fibrosis: a review. Biomolecules 11:789

14. Lindsey JD, Kashiwagi K, Boyle D, Kashiwagi E, Firestein GS, Weinreb RN (1996) Prostaglandins increase proMMP-1 and proMMP-3 secretion by human ciliary smooth muscle cells. Curr Eye Res 15(8):869-875

15. Lindsey JD, Kashiwagi K, Kashiwagi F, Weinreb RN (1997) Prostaglandins alter extracellular matrix adjacent to human ciliary muscle cells in vitro. Invest Ophthalmol Vis Sci 38:2214-2223

16. Ocklind A (1998) Effect of Latanoprost on the extracellular matrix of the ciliary muscle. A study on cultured cells and tissue sections. Exp Eye Res 67:179-191

17. Zhao F, Gandorfer A, Haritoglou C et al (2013) Epiretinal cell proliferation in macular pucker and vitreomacular traction syndrome: analysis of flatmounted internal limiting membrane specimens. Retina 33:77-88

18. Kamada R, Iwase T (2021) Rapid morphological restoration of normal foveal contour after spontaneous epiretinal membrane separation 
from retina in eye with macular pseudohole: a case report. Int Med Case Rep J 14:211-214. https://doi. org/10.2147/IMCRJ.S301252

19. Berufsverband der Augenärzte Deutschlands e.V. (BVA), Deutsche Ophthalmologische Gesellschaft (DOG), Retinologische Gesellschaft e.V. (RG) (2021) Stellungnahme von BVA, DOG und RG zur Entwicklung, Diagnostik und Behandlung der epiretinalen Gliose. Klin Monbl Augenheilkd 238(2):193-208. https://doi.org/10.1055/a-13518758. German. Epub 2021 Feb 22.PMID:33618391

\section{Aktuelle Buchempfehlungen aus dem Springer-Verlag}

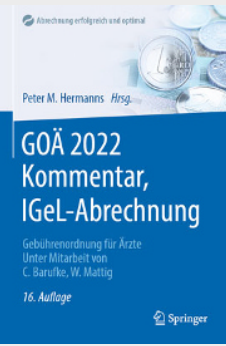

GOÄ 2022 Kommentar, IGeL-Abrechnung

\section{Gebührenordnung für Ärzte}

Hermanns, Peter M. (Hrsg.)

XXV, 896 Seiten

2022, 16. Auflage

Springer-Verlag

ISBN 978-3-662-64485-0

$79,99 €$

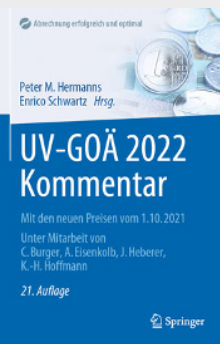

\section{UV-GOÄ 2022 Kommentar}

\section{Mit den neuen Preisen vom 1.10.2021}

Hermanns, Peter M., Schwartz, Enrico (Hrsg.)

XVII, 739 Seiten

2022, 21. Auflage

Springer-Verlag

ISBN 978-3-662-64487-4

$69,99 €$

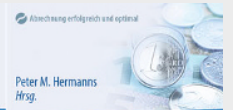

\section{EBM 2022 Kommentar}

Hermanns, Peter M. (Hrsg.)

XXVIII, 1004 Seiten

EBM 2022

Kommentar

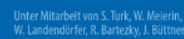

CBarnters

2022, 11. Auflage

Springer-Verlag

ISBN 978-3-662-64481-2

$79,99 €$

@ي Springer

EBM 2022

Kommentar

Kinderheilkunde

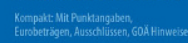

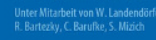

3. Aurfoge פิSpringer

\section{EBM 2022 Kommentar Kinderheilkunde}

Kompakt: Mit Punktangaben, Eurobeträgen, Ausschlüssen, GOÄ Hinweisen

Hermanns, Peter M. (Hrsg.)

XVII, 375 Seiten

2022, 3. Auflage

Springer-Verlag

ISBN 978-3-662-64483-6

$44,99 €$ 